\title{
Target Detection, Image Analysis, and Modeling
}

\author{
Mark E. NeLson
}

\section{Introduction}

One of the selective advantages afforded by the electric sense is that it enables animals to detect objects in dark aquatic environments where visual cues are limited. This ability, referred to as electrolocation, has functional similarities to the echolocation ability of bats and opens up a rich ecological niche for hunting at night or in turbid waters. Electrolocation also has functional similarities to visual processing because target objects form electrosensory images on a spatially distributed sensor array. This chapter summarizes recent advances in understanding target detection, localization, and image analysis in the electrosensory system, including contributions of mathematical modeling and computer simulation in helping to advance knowledge in this area.

Conceptually, the electrolocation task can be subdivided into three components-detection, characterization, and localization. The detection problem involves a determination of whether or not a potential electrolocation target is in the vicinity. In its simplest form, this can be formulated as a binary hypothesistesting problem, namely one that can be answered with either a "yes" or "no" response. If a potential target is present, the next conceptual stage is one of characterization. This stage requires an assessment of various target properties that can vary along a continuum, such as target size, shape, and electrical impedance. From a signal processing perspective, this is a problem in parameter estimation. If the behavioral response requires a directed movement, such as prey capture, then the final component of the task is one of localization. Localization involves estimation of quantities that can vary along a continuum, such as direction and distance to the target. More generally, the localization problem is a target-tracking task that may require estimation of dynamic properties of both the fish and prey, such as relative velocity and acceleration. Although the processes of detection, characterization, and localization have been described sequentially, they are tightly coupled and are likely to take place in parallel in the nervous system.

Electrolocation can be mediated by both low-frequency (Wilkens, Chapter 9) 
and high-frequency (Hopkins, Chapter 10) electrosensory systems. For animals that lack an electric organ, passive electrolocation is based on the ability to detect the intrinsic electric fields associated with target objects, such as bioelectric fields generated by prey. In contrast, animals that can generate their own electric field can detect a larger variety of target objects because active electrolocation requires only that the electrical properties of the target differ from those of the surrounding water. Aside from the source of the electric field, the functional principles of electrolocation are similar for both active and passive modes. To streamline the presentation, this chapter focuses on active electrolocation (reviewed in Moller 1995; von der Emde 1999; Bastian 2003). Comparisons between active and passive electrolocation are discussed in Nelson et al. (2002) and comparisons with the mechanosensory lateral line system can be found in this volume (Coombs, Chapter 12).

In the late 1950s, Lissmann and Machin (1958) first demonstrated that weakly electric fish (Gymnarchus niloticus) can detect and discriminate objects based solely on their electrical properties. They employed a clever experimental design in which artificial targets were placed inside visually opaque but electrically transparent porous pots. Using this approach they determined, for example, that Gymnarchus could detect the presence of a small glass rod (threshold diameter, $2 \mathrm{~mm}$ ) hidden inside a pot using only electrosensory cues. Generalizing from these early results and subsequent experiments using artificial targets, it has long been presumed that weakly electric fish, which are largely nocturnal and found in turbid waters, use electrolocation to hunt for food. Only recently have behavioral studies demonstrated that the electrosensory system is indeed involved in the detection and localization of small live prey (Wilkens et al. 1997; von der Emde and Bleckmann 1998; Nelson and MacIver 1999). This chapter focuses on prey capture as a prototypical electrolocation task that is linked to the animal's natural behavior and sensory ecology.

We begin, in Section 2, with an overview of the physics of active electrolocation and electrosensory image formation. Section 3 discusses the encoding of information by primary electrosensory afferent nerve fibers. Section 4 reviews key aspects of central neural processing, with an emphasis on the first stage of central processing in the hindbrain electrosensory lateral line lobe (ELL). Finally, Section 5 provides a summary of key insights that have been gained from studies of electrolocation and indicates directions for future research.

\section{Physics of Active Electrolocation}

This section provides an overview of the physics of electric field generation and electrosensory image formation. Physics-based models and computer simulation techniques can be combined to generate accurate representations of the fish's self-generated electric field and the electrosensory images that are formed on the skin when an object is placed in the field. Combining these modeling tech- 
niques with movement trajectories from three-dimensional video analysis allows the reconstruction of dynamic electrosensory images of prey capture events. This type of analysis provides a first glimpse of what the world "looks like" when viewed through an electric sense.

\subsection{Temporal Aspects of the Fish's Electric Field}

A natural starting point for describing the physics of active electrolocation is a description of the electric field that the fish uses to probe its environment. The electric field generated by a weakly electric fish can be characterized in terms of the temporal properties of the electric organ discharge (EOD) waveform and the spatial properties of the field surrounding the fish's body. The temporal properties of the EOD can be qualitatively assessed by placing a pair of wires in the water near the fish and listening to the EOD signal on an audio monitor. The EOD patterns of weakly electric fish fall naturally into two categories. Pulse-type fish generate brief discharges separated by significantly longer gaps, such that the audio output sounds like a series of clicks. Wave-type electric fish generate EODs with little or no gap between successive discharges, resulting in a periodic signal that sounds like a tone or hum.

Quantitative comparisons of EOD waveforms are typically made by recording the potential difference between the head and tail of the fish. Figure 11.1 illustrates head-tail waveforms for representative pulse-type and wave-type EODs, along with their corresponding power spectra. Because of inherent tradeoffs in time-frequency representations, pulse fish have EOD waveforms that are narrow in the time domain and broad in the frequency domain, while wave fish EODs are broad in the time domain and narrow in the frequency domain. Commonly studied species with pulse-type EODs include most African mormyrids, such as Gnathonemus petersii (elephant nose fish), and certain South American gymnotiform knifefish, including Gymnotus carapo (banded knifefish) and Brachyhypopomus pinnicaudatus. Commonly studied species with wave-type EODs include many other South American gymnotiform knifefish, such as Apteronotus albifrons (black ghost knifefish), Apteronotus leptorhynchus (brown ghost knifefish), Eigenmannia virescens (glass knifefish), and Sternopygus macrurus (longtail knifefish), as well as a single African mormyriform with a wave-type EOD, Gymnarchus niloticus (Aba aba) (Bass 1986; Zupanc and Bullock, Chapter 2).

The EOD amplitudes of both pulse and wave-type fish seem to remain constant during electrolocation behavior. Although there can be circadian, seasonal, and socially mediated changes in EOD amplitude, short-term modulations of EOD amplitude in association with electrolocation have not been reported. This is in contrast to some echolocating bats, which are known to decrease the intensity of their ultrasonic calls as they approach a prey target. This sort of intensity compensation behavior has not been observed in weakly electric fish. However, there are technical difficulties in making accurate measurements of 

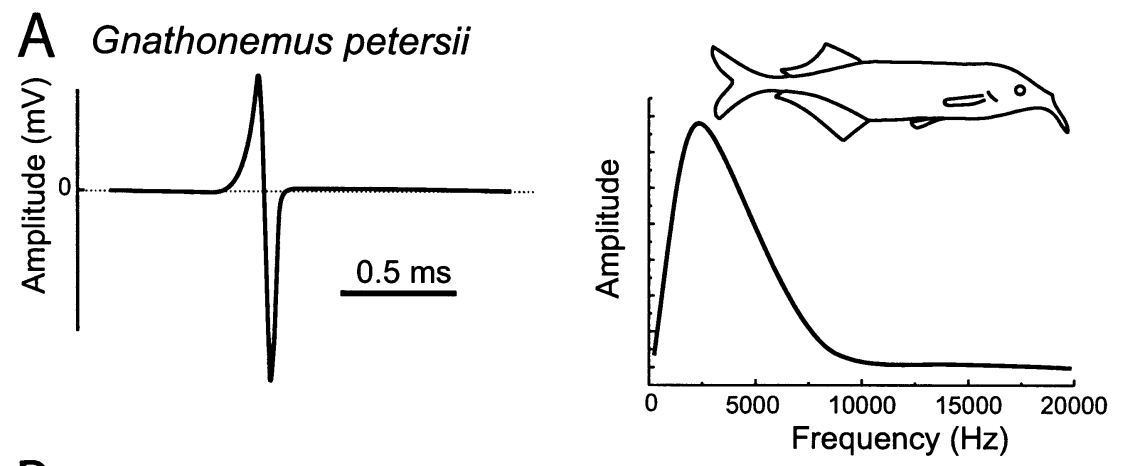

B Eigenmannia sp.
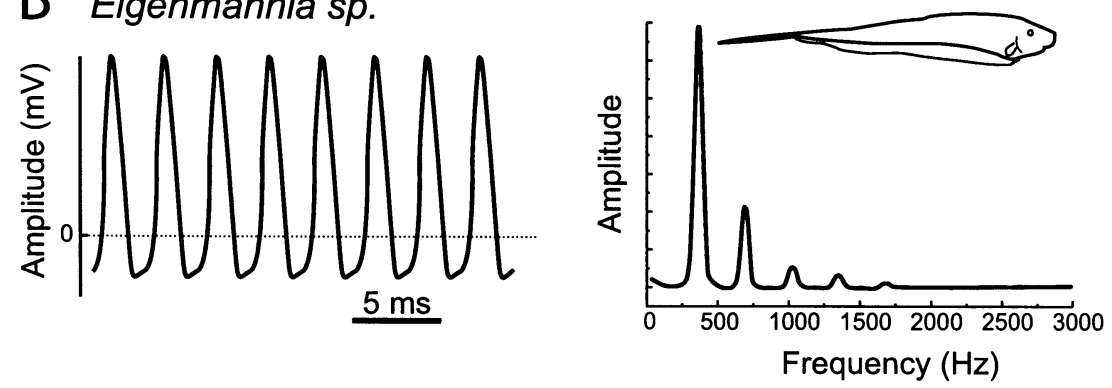

FIGURE 11.1. Electric organ discharge (EOD) waveforms (left) and power spectra (right) for weakly electric fish with pulse-type (A) and wave-type (B) discharges. (A) The pulsetype mormyrid Gnathonemus petersii generates brief pulses that have a broad frequency spectrum. (B) The wave-type gymnotiform Eigenmannia sp. generates a quasi-sinusoidal waveform with a frequency of a few hundred Hertz with most of the power concentrated at the fundamental frequency. Note that the time and frequency scales are different in (A) and (B). (From von der Emde 1999, with permission.)

EOD amplitude in free-swimming fish, so stringent tests of the constantamplitude hypothesis have not been made.

Wave-type fish also seem to maintain a constant EOD frequency during electrolocation behavior, whereas pulse-type fish can alter the EOD repetition rate. In fact, the short-term stability of EOD frequency in wave-type fish places it among the most regular known biological oscillators (Moortgat et al. 1998). Changes in EOD frequency can be induced by environmental factors as well as a variety of social stimuli, but frequency modulation does not appear to play a role in electrolocation behavior. Pulse-type fish, on the other hand, often exhibit increases in EOD rate and regularity in response to detection of a novel electrolocation target. In some ways this is reminiscent of echolocating bats that increase the frequency of their calls as they approach a prey target, culminating in a so-called "feeding buzz." However, changes in EOD rate in pulse fish seem to reflect a more general increase in electrosensory arousal, rather than target- 
specific modulations (reviewed in Moller 1995). The functional equivalent of a "feeding buzz" has not been reported in pulse fish. For further discussion of factors that can influence EOD properties, see Macadar et al. (Chapter 14).

\subsection{Spatial Aspects of the Fish's Electric Field}

In contrast to the heterogeneity of EOD temporal properties, the spatial structure of the electric field is similar for all weakly electric fish, including both waveand pulse-type species. This is a consequence of the physics of electric field generation, which results in a roughly dipolar field pattern surrounding the fish, independent of the temporal structure of the EOD waveform. Figure 11.2 shows a contour map of the empirically measured electric potential in the horizontal midplane of A. albifrons (Knudsen 1975). Electric potentials are always reported relative to some reference value. This is analogous to elevations on a topographic map being reported relative to sea level. When visualizing spatial maps of the electric field, the most common convention is to report potentials relative to a remote reference.

The contour levels shown in Figure 11.2 represent the amplitude of the EOD

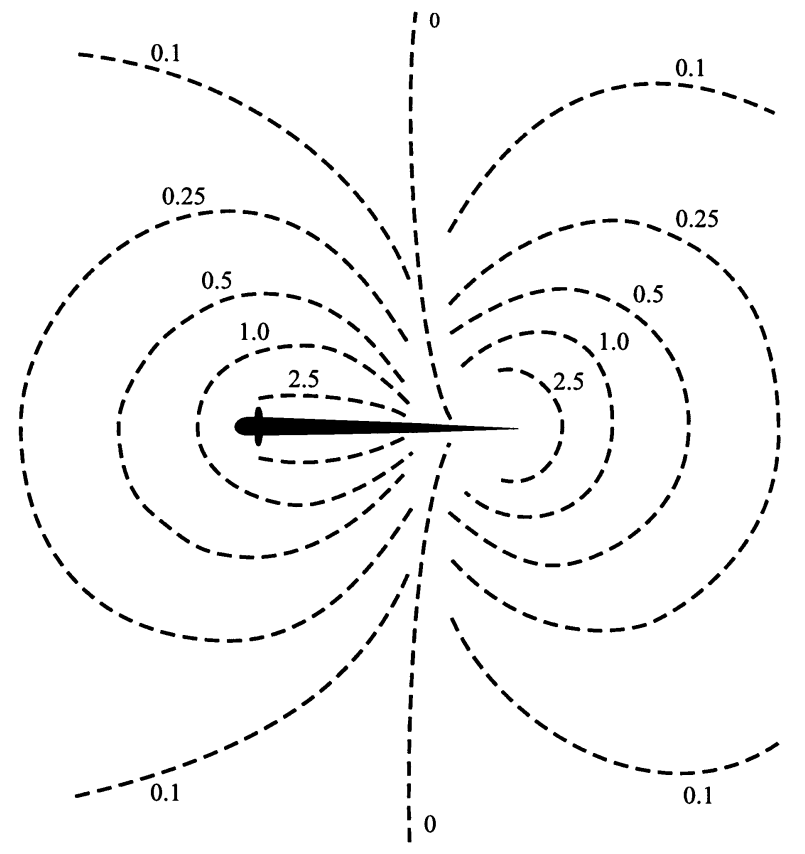

FIGURE 11.2. Equipotential lines (dashed line) illustrating the spatial structure of the electric field surrounding a $22 \mathrm{~cm}$ long Apteronotus albifrons. Potentials are reported as the peak-to-peak amplitude in microvolts relative to a remote reference. (Redrawn from Knudsen 1975, with permission.) 
waveform at different points in space. Note that the peak-to-peak values are always positive. If, however, the EOD had been "frozen" at a single instant in time, say when the head region was at the positive peak of the EOD waveform, then the isopotential values in the tail region would have had the opposite polarity from those in the head region.

To a first approximation, the spatial structure of the electric field surrounding an electric fish can be described as a dipole field, with an intensity that varies in proportion to the instantaneous amplitude of the EOD. Figure 11.3 shows an ideal current dipole, consisting of a current source of magnitude $+I$ at location $\mathbf{p}_{+}$and a current sink of magnitude $-I$ at $\mathbf{p}_{-}$in an infinite volume conductor of conductivity $\sigma$. At an arbitrary point $\mathbf{p}$ in three-dimensional space, the electric potential $\phi(\mathbf{p})$ is given by:

$$
\phi(\mathbf{p})=\frac{I}{4 \pi \sigma}\left(\frac{1}{\left|\mathbf{p}-\mathbf{p}_{+}\right|}-\frac{1}{\left|\mathbf{p}-\mathbf{p}_{-}\right|}\right)=\frac{I}{4 \pi \sigma}\left(\frac{1}{\mathbf{r}_{+}}-\frac{1}{\mathbf{r}_{-}}\right)
$$

A useful approximation can be derived when the distance $r$ from the point $\mathbf{p}$ to the center of the dipole is much greater than the separation between the two poles $d$. This case $(r>>d)$ is known as the far-field approximation. In the far field, the dipole potential is given by:

$$
\phi(r, \theta)=\frac{I d \cos \theta}{4 \pi \sigma r^{2}}
$$

The electric field vector $\mathbf{E}$ is given by the spatial gradient of the potential $\phi$ (Jackson 1999):

$$
\mathbf{E}=-\nabla \phi
$$

For a dipole field, it is convenient to specify the electric-field vector in spherical coordinates. In spherical coordinates, $r$ and $\theta$ are as shown in Figure 11.3, and the third dimension $\varphi$ (not shown) represents the rotational angle out of the

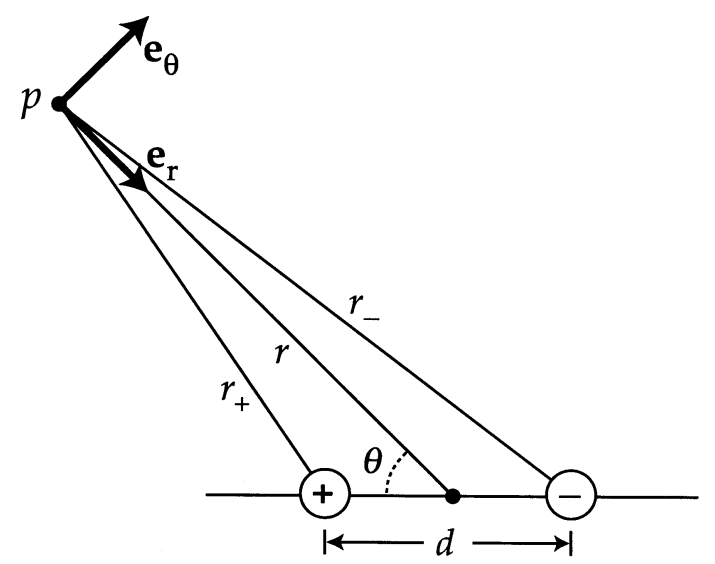

FIGURE 11.3. Coordinate system and definitions for calculating the electric potential at an arbitrary point $p$ in threedimensional space arising from an ideal current dipole. See text for explanation. 
plane of the figure. In this coordinate system, the gradient operator is defined by:

$$
\nabla \phi=\frac{\partial \phi}{\partial r} \hat{\mathbf{e}}_{r}+\frac{1}{r} \frac{\partial \phi}{\partial \theta} \hat{\mathbf{e}}_{\theta}+\frac{1}{r \sin \varphi} \frac{\partial \phi}{\partial \varphi} \hat{\mathbf{e}}_{\varphi}
$$

Combining Equations (2) to (4), the $\mathbf{E}$ vector in the far field is given by:

$$
\mathbf{E}(r, \theta)=\frac{I d}{4 \pi \sigma r^{3}}\left(2 \cos \theta \hat{\mathbf{e}}_{r}+\sin \theta \hat{\mathbf{e}}_{\theta}\right)
$$

where $\hat{\mathbf{e}}_{r}$ and $\hat{\mathbf{e}}_{\theta}$ are unit vectors in the radial and tangential directions, as shown in Figure 11.3. Because of the cylindrical symmetry about the axis of the dipole, there is no gradient, and hence no electric field component, in the azimuthal direction $\hat{\mathbf{e}}_{\varphi}$ (which would be pointing out of the plane of Figure 11.3).

At discharge frequencies relevant to electrolocation (see Fig. 11.1), the electrical properties of the water can be treated as purely resistive. In this case, the current density in the water $\mathbf{J}$ is related to the electric field $\mathbf{E}$ by $\mathbf{J}=\sigma \mathbf{E}$, which can be viewed as a form of Ohm's law. Thus electric current flows in the direction of the local $\mathbf{E}$ vector. Because $\mathbf{E}$ is the gradient of the potential, the $\mathbf{E}$ vectors are oriented perpendicular to the isopotential lines, as shown in Figure 11.4. Going back to the topographic map analogy, electric current flows downhill along the path of steepest descent.

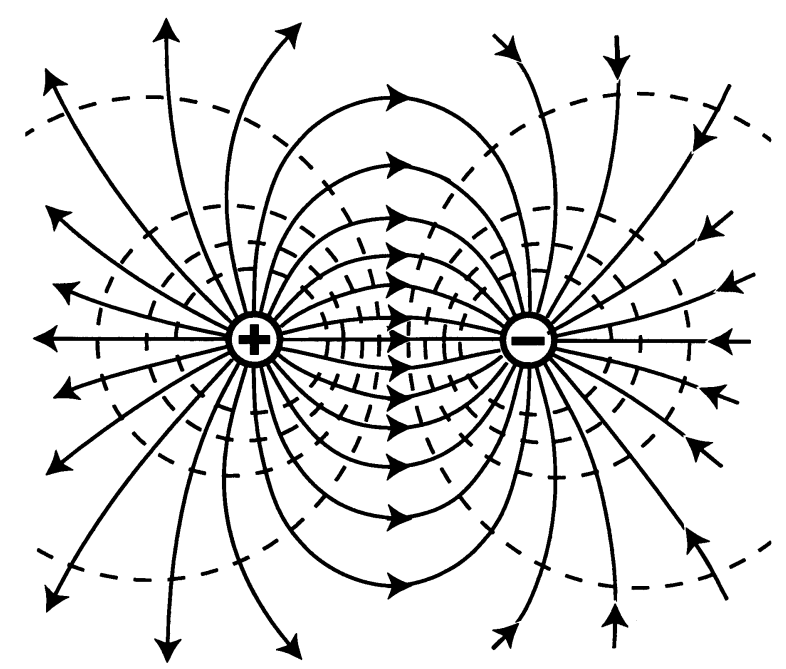

FIGURE 11.4. Illustration of the lines of current flow (solid lines) associated with a dipole field. The direction of current flow is orthogonal to the isopotential contours (dashed lines), and current flow is from positive to negative. 


\subsection{Properties of the Near Field}

The effective range of electrolocation for detecting small prey is typically less than the fish's body length (MacIver et al. 2001). Hence, the relevant part of the field for understanding target detection is the "near field" rather than the far field. The far-field approximation described above is useful for developing a general understanding of the field structure. However, in the near field (less than a few body lengths), the spatiotemporal structure becomes more complex than predicted by a simple dipole model. One indication of this is shown in Figure 11.2, where it can be seen that the isopotentials near the head and trunk are elongated in the direction of the fish's body axis compared to the corresponding isopotentials in the tail region. This arises because the relatively low internal body resistance of the fish effectively extends the poles, making them appear as line sources rather than point sources. Another important effect in the near field is that the power-law scaling is different. In the far field, the magnitude of the electric field falls with distance as $r^{-3}$ (Eq. 5). In the near field, the attenuation with distance cannot be accurately described by a power law with a constant exponent. In general, the field intensity falls off more slowly in the near field than in the far field. Fits to empirical data yield approximate power law attenuation near the body of $r^{-1}$ to $r^{-2}$ depending on location (Rasnow and Bower 1996). Recently, detailed maps of the near field region have been constructed at multiple time points during the EOD cycle for several different species (reviewed in Assad et al. 1999). These dynamic maps reveal interesting spatiotemporal structure that is not evident in the static snapshot shown in Figure 11.2. These empirical maps provide the most detailed and reliable descriptions of the spatiotemporal properties of the near field.

\subsection{Target Perturbations and Image Formation}

The key principle of active electrolocation is based on the fact that an object that differs in electrical impedance from the surrounding water can perturb the flow of electric current near the fish. These perturbations give rise to changes in the local potential difference across the skin of the fish, referred to as the transdermal voltage. For example, a nonconducting object near the fish would block the flow of electrical current, causing the current lines to flow around the object as shown in Figure 11.5. This decrease in current density causes a localized decrease in voltage drop across the skin. Thus, a nonconducting object casts a sort of electrosensory "shadow" on the surface of the fish. Conversely, a conducting object would increase the local current density, and hence increase the local transdermal voltage, giving rise to an electrosensory "bright spot" on the surface of the fish. Thus, objects near the fish that differ in conductivity from the surrounding water cause local modulations of the transdermal voltage. Electrosensory images correspond to changes in transdermal voltage on the electroreceptor surface of the skin, just as visual images correspond to changes in 


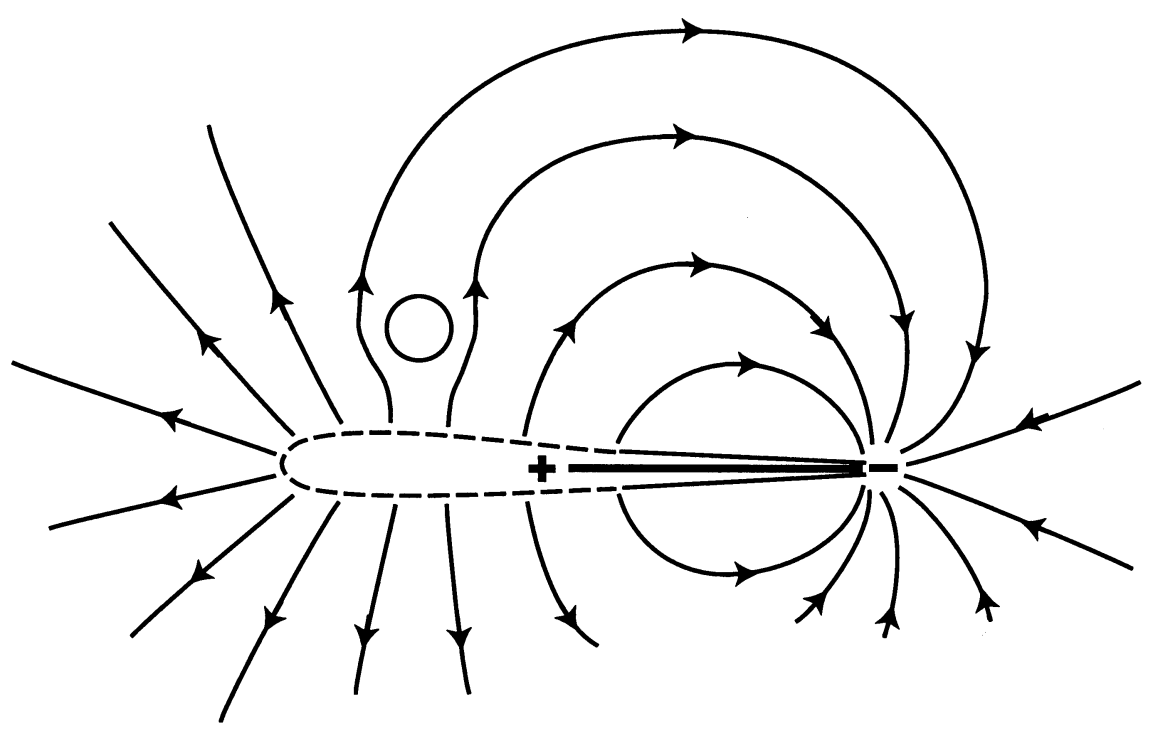

FIGURE 11.5. Schematic representation of the principles of active electrolocation. The electric organ (solid black bar) gives rise to a dipolar field pattern around the fish. The electric current follows the local field lines (solid lines with arrows). A nonconducting target object (circle) perturbs the flow of electric current, causing a local decrease in current density near the object. This decrease in current density translates into a decrease in the transdermal voltage across the skin near the object. The spatial pattern of the transdermal voltage across the sensory surface represents the electric image of the object. (Redrawn from Heiligenberg 1977, with permission.)

light intensity on the photoreceptor surface of the retina. Since there is no functional equivalent to the lens of the eye, electrosensory images are blurred and the degree of blur increases with target distance.

The electrosensory image of a target object depends on multiple factors, including target properties (location, size, shape, and electrical impedance), fish properties (amplitude and spectral characteristics of the fish's electric field at the target location), and environmental properties (water conductivity, background noise characteristics). Some electrosensory targets, such as small prey, can be well approximated as spherical targets. The analytic formula for the voltage perturbation caused by a perfectly conducting sphere of radius $a$ is (Rasnow 1996):

$$
\delta \phi(\mathbf{r})=\frac{a^{3} \mathbf{E}_{\mathrm{f}} \cdot \mathbf{r}}{r^{3}}=\frac{a^{3} E_{\mathrm{f}} \cos \theta}{r^{2}}
$$

This equation, like Equation (2), is also the equation of a dipole potential. When a small spherical target is placed in an electric field, a dipole perturbation is induced at the location of the target (Jackson 1999). In this equation, $\delta \phi(r)$ 
represents the change in potential at position $r$ relative to the center of the target object. The $a^{3}$ term is proportional to the volume of the target. This is an important result for predicting the effects of differently sized targets. Doubling the radius of a target will produce an eightfold increase in the voltage perturbation. When computing electrosensory images, $r$ will be a vector from the target object to a point on the fish's sensory surface. The term $\mathbf{E}_{\mathrm{f}}$ represents the fish's electric field vector at the location of the target object. The voltage perturbation $\delta \phi$ scales linearly with the strength of the fish's field at the target. The perturbation is strongest when the direction of the fish's electric field vector $\mathbf{E}_{\mathrm{f}}$ is aligned with the direction of the $\mathbf{r}$ vector (i.e., $\cos \theta=1$ ). Over much of the head and trunk region of the fish, the $\mathbf{E}$-vector is approximately normal to the body surface. Hence, the peak perturbation tends to occur at a point on the surface directly "underneath" the target object. Away from this peak, the decrease in voltage perturbation gives rise to a roughly Gaussian shaped electrosensory image on the sensory surface. Representative electrosensory images are shown in Figure 11.6A.

Equation six specifies the voltage perturbation for a perfect conductor. The more general case takes into account the resistivity and dielectric properties of the target and the surrounding water (Rasnow 1996):

$$
\delta \phi(\mathbf{r})=\frac{\mathbf{a}^{3} \mathbf{E}_{\mathrm{f}} \cdot \mathbf{r}}{r^{3}}\left[\frac{\left(\rho_{w}-\rho_{t}\right)-i \bar{\omega} \rho_{w} \rho_{t}\left(\varepsilon_{w}-\varepsilon_{t}\right)}{\rho_{w}+2 \rho_{t}+i \bar{\omega} \rho_{w} \rho_{t}\left(2 \varepsilon_{w}+\varepsilon_{t}\right)}\right]
$$

where $\rho_{w}$ is the water resistivity, $\rho_{t}$ is the target resistivity, $\varepsilon_{w}$ is the dielectric constant of water, $\varepsilon_{t}$ is the dielectric constant of the target, $\omega=2 \pi f$ is the angular frequency of the fish's electric field and $i=\sqrt{-1}$. There are several interesting features to note about this relationship. The term in square brackets on the right hand side of Equation (7) represents the electrical contrast $\chi$ of the target:

$$
\chi=\frac{\left(\rho_{w}-\rho_{t}\right)-i \bar{\omega} \rho_{w} \rho_{t}\left(\varepsilon_{w}-\varepsilon_{t}\right)}{\rho_{w}+2 \rho_{t}+i \bar{\omega} \rho_{w} \rho_{t}\left(2 \varepsilon_{w}+\varepsilon_{t}\right)}
$$

If the resistivity and dielectric constant of the target match that of the water, then the numerator is zero, and the electrical contrast $\chi=0$, which is to say that the target becomes electrically invisible. For a perfect conductor $\left(\rho_{t}=0\right)$, the electrical contrast is unity $\chi=1$. Metal spheres closely approximate a perfect conductor and are often used as targets in electrolocation experiments. For many insulating materials, such as rocks, air bubbles, or plastic spheres, the resistivity of the target is much higher than that of the surrounding water $\left(\rho_{t}\right.$ $\left.>>\rho_{w}\right)$ and the dielectric constant is significantly less than water $\left(\varepsilon_{r}<<\varepsilon_{w}\right)$. In this case, Equation 8 simplifies to $\chi=-1 / 2$.

The electrical contrast is a complex number, with both real and imaginary parts. The real part is related to the resistive component of the target impedance, while the imaginary part is related to the target capacitance. Note that the imaginary parts of Equation 8 are proportional to the frequency of the fish's electric field. In general, fish with higher frequency components in their EOD 
A Transdermal Potential Change B Afferent Firing Rate Change
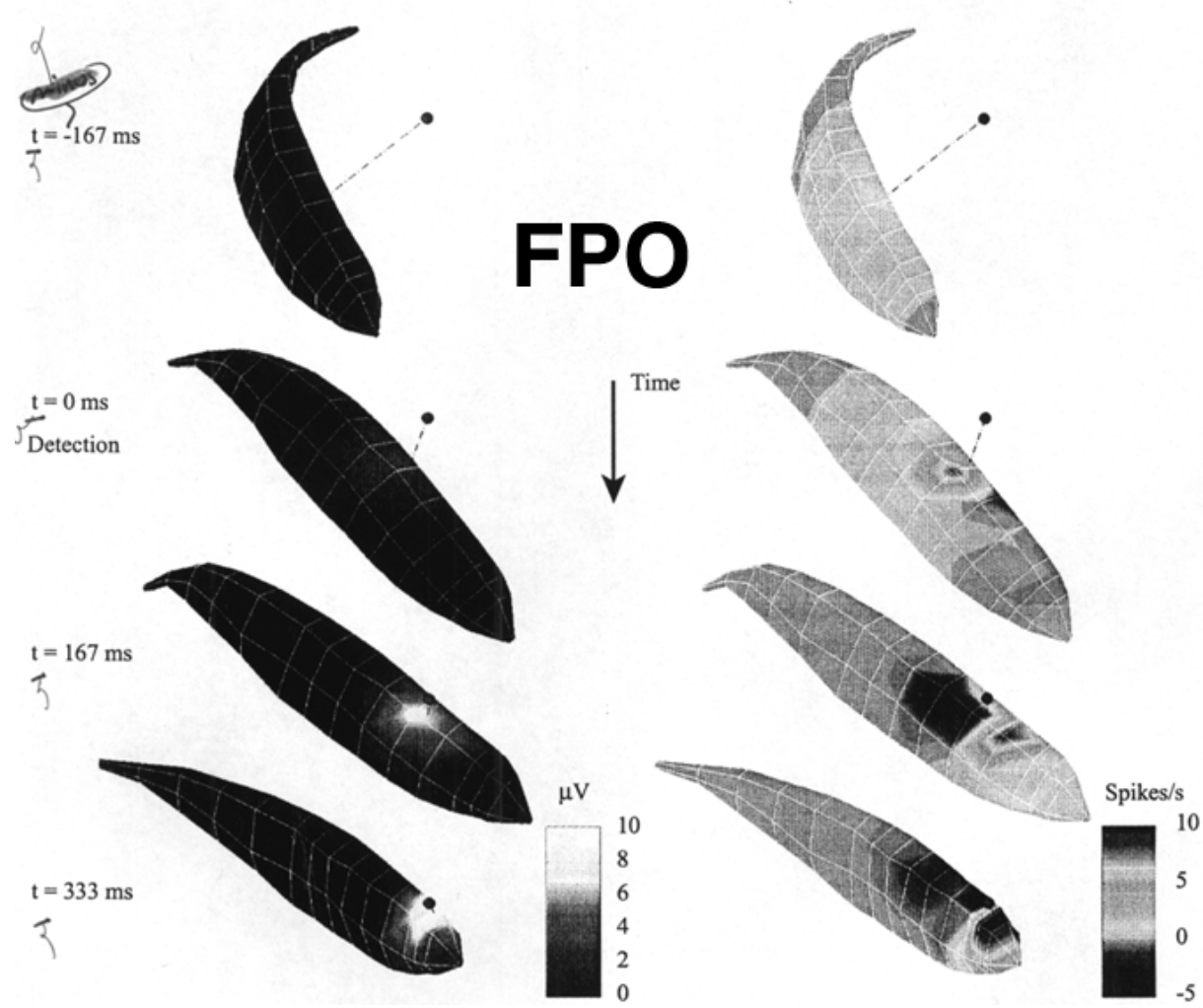

FIGURE 11.6. Reconstructed electrosensory images from a prey capture study. Each column shows snapshots of the model fish and prey at four different times in the prey capture sequence. The left-hand column (A) shows the change in transdermal voltage induced by the prey (small sphere). The right-hand column (B) shows the predicted change in spike activity on P-type primary electrosensory afferent nerve fibers. The dashed line indicates the shortest distance between the fish and prey. (From MacIver and Nelson 2000, with permission.)

waveform are better able to probe the capacitive components of object impedance. Both pulse-type and wave-type fish have been shown to be able to detect capacitive components of target impedance (reviewed in von der Emde 1999). Pulse-type fish appear to accomplish this by analyzing changes in the EOD waveform, while wave fish are thought to analyze timing shifts between parts of the body at different relative distances from the target (von der Emde 1999).

\subsection{Modeling the Electric Field and Electrosensory Images}

Several approaches have been used for modeling the spatial pattern of the electric field surrounding the fish and the image properties of electrolocation targets.

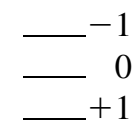


Seminal work by Heiligenberg (1975) used a two-dimensional finite-difference technique to numerically solve for the electric potential in the horizontal midplane of the fish. This work led to new insights regarding the importance of fish body geometry and impedance characteristics in electrolocation performance. Hoshimiya et al. (1980) extended this approach using a higher resolution finite-element method. Bacher (1983) introduced an analytic technique that treated the electric organ as an extended line charge. Caputi et al. (1998) used a finite difference technique constrained by empirical measurements of skin resistance to accurately describe electrosensory images of resistive targets in pulse fish and extended these results to include objects with complex impedance (Budelli and Caputi 2000). Assad and colleagues have used a three-dimensional boundary element technique to accurately model both spatial and temporal aspects of the field (Assad 1997). Over the years, increasingly sophisticated simulation techniques have led to increasingly accurate models of the electric field. These electric field models provide a foundation for experimental and theoretical studies of electrosensory image formation and neural processing.

\subsection{Spatiotemporal Properties of Dynamic Targets}

Relative motion between the fish and prey converts a static spatial image into a dynamic spatiotemporal pattern. For example, if an electrosensory image with a spatial width of $1 \mathrm{~cm}$ on the skin sweeps across the sensory array at a velocity of $10 \mathrm{~cm} / \mathrm{s}$ (a typical swimming velocity), then an individual receptor situated along the projected path of the target would experience an activation profile with a temporal width of $0.1 \mathrm{~s}$. Spatiotemporal relationships are more complex during prey capture behavior because target distance and relative velocity are constantly changing. Figure 11.6A shows snapshots of computer-reconstructed electrosensory images that occur during prey capture behavior of A. albifrons (Nelson and MacIver 1999). Initially, when the prey is several centimeters from the fish, the electrosensory image is weak and diffuse (Fig. 11.6A, top). The image becomes progressively stronger and narrower as the relative distance between the fish and prey decreases. Just prior to capture, the image sweeps across the head at a distance of just a few millimeters, giving rise to intense, focal images (Fig. 11.6A, bottom). These dynamic changes in spatiotemporal image properties occur over a time course of approximately $500 \mathrm{~ms}$, from the time of initial prey detection to the final capture.

\subsection{Motor Strategies Associated with Electrolocation}

Motor control of body position and swimming velocity can actively influence the spatiotemporal properties of electrosensory images. Weakly electric fish have been observed to use a variety of motor strategies while exploring their environment, including tail-probing, body bends, back-and-forth scanning, reverse swimming, and body rolls (see Hopkins, Chapter 10). These motor behaviors affect the quality and content of incoming sensory signals, in much the same way that head and eye movements influence visual input. Knifefish are 
particularly remarkable in this regard because of the high degree of maneuverability afforded by a ribbon fin propulsion system. Knifefish can swim both forwards and backwards with a broad range of velocities and body orientations.

When weakly electric knifefish capture prey, they usually do so by swimming backwards (Lannoo and Lannoo 1993; MacIver et al. 2001). During the search phase, prior to detection, the fish is often swimming forwards. When a target is detected, typically at a distance of a few centimeters for small prey (MacIver et al. 2001), the fish executes a rapid reversal in swimming direction and backs up to capture the target, as shown in Figure 11.6. In addition to backing up, the fish executes body movements that bring the sensory surface closer to the target. In A. albifrons, the receptor organ density in the head region is about 5 to 10 times higher than on the trunk (Carr et al. 1982). Functionally, the head region can be considered as an "electrosensory fovea." As the fish executes movements to bring the target toward this high-acuity area, the relative distance between the target and the sensor array decreases with time, thereby providing the fish with a progressively stronger and sharper electrosensory image of the target.

The backward swimming capability of gymnotiform knifefish has been a subject of much discussion and speculation in the literature. Ribbon fin propulsion effectively decouples control of locomotion from trunk movements. Lissman (1958) and others speculated that the ability to locomote while maintaining a rigid body posture might help electric fish avoid electrosensory reafference that would be caused by tail movements during swimming. However, video analysis of feeding behavior in A. albifrons has shown that body bends are a common feature of prey-capture behavior (MacIver et al. 2001), suggesting that maintaining a rigid trunk is not a key constraint on the animal's motor strategy. It is now known that neural circuitry in the hindbrain electrosensory nucleus is able to adaptively cancel out most of the reafferent components of the input signal at the first stage of central processing (see Bastian and Zakon, Chapter 8).

\subsection{Signal Strength and Signal-to-Noise Considerations}

There are two metrics to consider when assessing the strength of a sensory signal. One measures the signal strength relative to some absolute reference level, such as the animal's behavioral threshold in the absence of noise. The other metric measures signal strength relative to the context-dependent background noise, and is typically characterized by a signal-to-noise ratio (SNR). It is possible for a signal to be strong by one metric, but weak by the other. For example, the sound intensity of a soft whisper is about two orders of magnitude above the human auditory threshold, but a whisper can be impossible to detect in a noisy environment, such as a rock concert.

For the high-frequency electrosensory system, behavioral detection thresholds have been estimated to be on the order of $0.1 \mu \mathrm{V}$, when expressed as the root mean square (RMS) change in transdermal potential, or about $0.1 \mu \mathrm{V} / \mathrm{cm}$ when 
expressed as a field gradient in the water outside the skin (Rasnow 1996). This represents about a $0.1 \%$ change in the baseline transdermal potential or external field gradient. Based on reconstructed prey-capture events, MacIver et al. (2001) estimated the peak change in transdermal potential at the time of prey detection to be on the order of $1.0 \mu \mathrm{V}$ RMS, assuming the electrical contrast of the prey to be unity (i.e., a perfect conductor). Factoring in the actual electrical contrast of the prey drops this estimate to about $0.4 \mu \mathrm{V}$ RMS (Nelson et al. 2002). Thus, prey-related electrosensory signals are estimated to be only modestly above behavioral threshold at the time of detection. This implies that in the absence of noise the fish should be able to detect the prey using information from the highfrequency electric sense. If, however, the fish encounters significant noise in its natural environment, then this weak signal may be difficult to detect.

The SNR provides a measure of the strength of a signal relative to background noise. One significant source of background noise in the electrosensory system is associated with the fish's own movements. During swimming and exploratory movements, changes in the position of the trunk and tail will alter the position of the electric organ relative to the electroreceptor array. Empirically measured tail bend modulations in A. leptorhynchus are on the order of 20 to $50 \mu \mathrm{V}$ RMS for tail bends in the range of 20 to $50^{\circ}$, or approximately $1 \mu \mathrm{V}$ RMS per degree (Bastian 1995). Based on the RMS value of tail bend angle during prey capture behavior (MacIver et al. 2001), the associated background noise is on the order of $30 \mu \mathrm{V}$ (RMS), resulting in a SNR $<<1$. Fortunately, tail bends are not purely random events and the effects can be predicted. Tail bends are initiated by outgoing motor commands and are monitored by proprioceptors. The nervous system therefore has access to efference copy and sensory feedback signals that are correlated with tail position (Bastian 1995). Neural circuitry in the electrosensory lateral line lobe (ELL) makes use of such signals to adaptively suppress the predictable component of the electrosensory background associated with tail bending (Bastian and Zakon, Chapter 8), thus greatly improving the effective SNR.

In addition to tail-bend modulations, several other sources of background noise can influence target detectability. Depending on the context, the target signal might be obscured by other objects in the electrosensory scene. This sort of background is often referred to as clutter. There is very little empirical information on natural electrosensory clutter or the properties of natural electrosensory scenes. In considering the problem of electrosensory clutter, there are several important points to keep in mind. (1) The electrosensory system is a short-range sense, so the electrosensory scene is dominated by objects that are within about one body length of the fish. This differs from the background clutter problem in long-range modalities such as vision and audition, in which sensory energy from distant objects constitutes a significant component of the background clutter. (2) There is likely to be significant spatial overlap in the images formed by different objects in the scene because electrosensory images are intrinsically blurred. (3) Nonconducting boundaries, such as the water surface or a large rock, can cause large-scale modulations over the entire body 
surface. The spatial filtering properties of neurons in the ELL may help the animal distinguish local modulations arising from small prey from the global modulations caused by nonconducting boundaries and other large objects in the electrosensory scene.

One additional source of background noise is associated with the sensory transduction process. Transdermal voltage changes are converted into membrane voltage changes in electroreceptor cells, which modulate the release of neurotransmitter and influence spike generation in primary afferent nerve fibers. At the biophysical level, each of these steps involves stochastic processes that potentially introduce noise into the measurement process. The next section discusses electroreceptor and primary afferent properties in more detail. It turns out that intrinsic variability in primary afferent spike trains can also contribute to the background noise that potentially limits the detectability of weak sensory signals.

\section{Neural Coding in Primary Electrosensory Afferents}

For the nervous system to carry out the computations necessary to support electrolocation, information about the transdermal voltage patterns on the skin must be converted into a neural representation. This conversion is performed by an array of electroreceptor organs that are embedded in the skin of the fish. Functionally, each electroreceptor organ acts as a sort of digital voltmeter, converting analog changes in transdermal voltage into trains of action potentials. The information needed for subsequent neural processing is encoded in the spike train data. The coding strategies implemented by these biological voltmeters are rather sophisticated, involving various forms of input filtering and noise suppression during the encoding process. Different classes of electroreceptor organs filter and encode the voltage data in different ways, providing the central nervous system (CNS) with an efficient representation for performing its computations. Electroreceptor afferents enter the CNS via the eighth cranial nerve and share similarities with afferents from other octavolateralis systems, including auditory, vestibular, and mechanosensory lateral line systems. This section describes some of the basic neural coding properties of primary electrosensory afferents, with an emphasis on amplitude coding in wave-type gymnotiforms. Anatomical, physiological, and biophysical properties of electroreceptors and primary afferents are discussed elsewhere in this volume (Northcutt, Chapter 5; Bodznick and Montgomery, Chapter 6, Kawasaki, Chapter 7).

\subsection{Types of Electroreceptor Organs and Primary Afferents}

Electroreceptor organs can be grouped into two broad categories based on their frequency filtering characteristics. Ampullary organs, which are found in all electroreceptive organisms, are sensitive to low-frequency electric fields (typically less than $50 \mathrm{~Hz}$ ). Ampullary organs are generally associated with passive 
electrolocation of extrinsic electric field sources. They also respond to lowfrequency components of the EOD associated with certain types of electrocommunication signals. Tuberous organs are found in all weakly electric fish and in the South American electric eel Electrophorus (which has both strong and weak EODs). Tuberous organs are sensitive to high-frequency (typically 0.1 to $10 \mathrm{kHz}$ ) electric fields, and typically tuned to frequencies near the peak of the EOD spectrum. Tuberous organs are generally associated with active electrolocation involving perturbations of the fish's own electric field. Tuberous organs also respond to electric fields generated by other electric fish, and thus play a role in electrocommunication and social interactions (Hopkins, Chapter 10; Jorgensen, Chapter 3). In general, tuberous receptor organs are broadly distributed over the entire body surface, often with a higher density in the head region. The total number of tuberous organs varies by fish size and species, but is typically in the range of several thousand.

Coding properties of electroreceptor organs are deduced from the spike activity of the electrosensory afferent nerve fibers that innervate them. In some cases, a single afferent fiber carries information from a single receptor organ, while in other cases, a single afferent pools information from multiple organs. It is also possible for a single electroreceptor organ to give rise to multiple afferent fibers. These relationships vary by species and by receptor subtype. The descriptions of coding properties given below generally apply to a "receptor unit" consisting of a single primary afferent nerve fiber and its corresponding set of one or more electroreceptor organs.

Fish with wave-type EODs have two subtypes of tuberous receptor units (Scheich et al. 1973). One type conveys information about stimulus amplitude, while the other type conveys information about stimulus timing. The ongoing quasi-sinusoidal oscillation of the fish's EOD provides a natural "clock" for the system. In wave-type fish, tuberous afferents fire at most one spike per clock cycle. Amplitude information is conveyed by a spike probability code. When no target object is present, probability-coding (P-type) units fire a spike with some baseline probability per EOD cycle, typically around 0.3 . When a conducting object approaches the receptor organ, it causes an increase in the local transdermal voltage and an increase in the per-cycle firing probability of the $\mathrm{P}$ unit. Similarly, when a nonconducting object approaches the receptor, the local transdermal potential and the P-unit firing probability will decrease. The interspike interval distribution is irregular, so P-type units are sometimes described as "sputtering." In contrast, time-coding (T-type) units fire exactly one spike per EOD cycle and are thus extremely regular. In a neurophysiological recording, the spike activity of a T-type unit sounds like a constant tone on an audio monitor. For T-type units, the spike time is tightly phase-locked to the EOD cycle. Local phase shifts in transdermal potential, as might be induced by the capacitive component of target impedance, will cause corresponding shifts in spike timing in T-type units. It is interesting to note that the only African mormyriform with a wave-type EOD, Gymnarchus, independently evolved the same electrosensory coding strategy as the South American wave gymnotiforms. 
Although the functional properties are very similar, the nomenclature is different in Gymnarchus; the functional equivalents of P-type and T-type units are called O type and S type, respectively. The segregation of amplitude and timing information is also a common feature of auditory processing, and many of the coding principles are similar for electrosensory and auditory systems (Carr 1986).

Fish with pulse-type EODs also extract information on amplitude and timing, but use a different coding strategy. Gymnotiform pulse species have pulse markers ( $\mathrm{M}$ units) that fire a single, short-latency spike following each EOD and are functionally similar to time coding units in wave species. Amplitude information is encoded by burst duration coders (B units), which convert stimulus intensity into bursts that range from one spike for a weak stimulus, to more than 10 spikes for a strong stimulus. Information about stimulus intensity is contained both in the burst count and in the latency to the first spike (Yager and Hopkins 1993). Mormyrids with a pulse-type EOD have two main classes of tuberous receptor units. One class (knollenorgan) is specialized for electrocommunication and is not considered further; the other class (mormyromast) is specialized for active electrolocation. Mormyromast afferents respond to each EOD with a short burst of one to three spikes. The intensity of the stimulus is encoded in both the latency to the first spike as well as the number of spikes in the burst. Mormyromast afferents typically operate somewhere in the middle of their dynamic range, such that their response can be modulated in both directions by targets of differing conductivity. Each mormyromast organ contains two types of separately innervated receptor cells. Both types encode stimulus amplitude, but one type is sensitive to small phase shifts that distort the EOD waveform, whereas the other is not (Bell 1990). Comparisons between responses of these two fiber types may allow the fish to assess the resistive and capacitive components of target impedance (reviewed in von der Emde, 1999).

\subsection{Information Coding Properties of P-Type Units}

P-type (probability coding) afferents of wave-type gymnotiforms have received considerable attention in terms of understanding their response dynamics and information coding capabilities and are therefore treated in more detail here. Under baseline conditions, with no target present, the input stimulus for a $\mathrm{P}$ unit is the local oscillatory transdermal potential created by the fish's own EOD. The amplitude of these oscillations will increase when a conducting object approaches and will decrease when a nonconducting object approaches. The baseline EOD oscillation serves as a carrier signal and a target object induces an amplitude modulation (AM) of this carrier signal. P-type units are tuned to the carrier frequency of the fish's own EOD, with a $\mathrm{V}$-shaped tuning curve (Hopkins 1976). This filtering helps improve the SNR by filtering out background electrical noise in frequency bands other than those close to the fish's own EOD that are functionally important for electrolocation and electrocommunication.

Another type of filtering takes place in $\mathrm{P}$ units that is related to the AM 
frequency tuning rather than the carrier tuning. The frequency content of an AM signal induced by a target is related to its speed and distance. Nearby, fastmoving targets will cause higher-frequency AMs compared to distant, slowmoving targets. P-type units act as high-pass filters in the AM frequency domain, giving the strongest response to fast components of the AM signals (Bastian 1981a; Nelson et al. 1997). If a target remains stationary relative to the fish, P-unit activity gradually adapts back to baseline firing with a multiscale relaxation time course (Xu et al. 1996). The high-pass AM filtering properties and adaptation characteristics of $\mathrm{P}$ units reflect the fact that changes in the local transdermal potential carry more behaviorally relevant information than the absolute magnitude of the transdermal potential. The high-pass filter characteristics can be seen in Figure 11.6B.

Another important aspect of P-unit coding is related to the statistical properties of the afferent spike trains. P-unit spike activity is relatively irregular on short time scales, which explains why they are often described as "sputtering." This short-term variability can be quantified by the coefficient of variation of the interspike interval (ISI) distribution, which is typically around 0.5. However, this measure characterizes variability only on short time scales, comparable to the mean ISI, which is on the order of a few milliseconds in A. albifrons. Although P-unit spike trains are irregular on short time scales, they show a remarkable degree of regularity on intermediate time scales of 100 to $200 \mathrm{~ms}$ (30 to 60 ISIs). Spike train regularization on this time scale significantly enhances the detectability of weak signals by decreasing the effective background noise from spike train fluctuations (Ratnam and Nelson 2000).

Information-theoretic measures have been used to demonstrate that P-type afferents accurately encode information about the temporal waveform of AM signals within the behaviorally relevant bandwidth. Under optimal conditions, the information that can be transmitted about a band-limited random AM signal is within a factor of two of the theoretical maximum. Under these conditions, the information content in the spike train is on the order of one bit per spike, resulting in overall information rates of several hundred bits per second (Gabbiani et al. 1996; Wessel et al. 1996; Gabbiani and Metzner 1999).

\subsection{Models of P-Unit Response Dynamics and Spike Train Statistics}

Over the last several years a number of P-type afferent models have been developed. Kashimori et al. (1996) presented detailed biophysical models of Pand T-type electroreceptor organs, but did not explicitly address issues of neural coding or information transmission. Nelson et al. (1997) developed a computational model of afferent dynamics that reproduced the AM filtering characteristics and short-term variability of P-unit spike trains. Kreiman et al. (2000) developed a similar model to explain their experimental data on cross-trial variability when P units are given repeated presentations of an identical "frozen noise" stimulus. Under baseline conditions, both the Nelson et al. (1997) and 
the Kreiman et al. (2000) models produce uncorrelated ISI sequences and hence do not accurately describe longer-term spike train regularization, which is important for improving weak signal detectability.

Chacron et al. (2000) developed a P-unit model that included a dynamic threshold that was transiently elevated following each spike to give rise to a relative refractory period. This model yields an ISI distribution that is consistent with experimental data and also reproduces correlations between neighboring intervals in the ISI sequence. An extended and more detailed version of this model is able to reproduce the frequency response characteristics of P-type afferents as well as the key features of long-term spike train regularization (Chacron et al. 2001). Brandman and Nelson (2002) developed a reduced version of the Chacron model that has been used to simulate afferent activity over the entire population of 15,000 $\mathrm{P}$ units in A. albifrons during reconstructed prey capture sequences, producing dynamic spatiotemporal images similar to those shown in Figure 11.6B.

\section{Central Information Processing}

The first stage of information processing in the CNS takes place in the hindbrain electrosensory lateral line lobe (ELL). The ELL is the sole recipient of electrosensory primary afferent input and thus all electrosensory information needed to support electrolocation passes through this structure. Detailed anatomical and physiological descriptions can be found elsewhere in this volume (Bell and Maler, Chapter 4; Kawasaki, Chapter 7). The ELL has structural and functional similarities to the dorsal cochlear nucleus in the mammalian auditory system (Montgomery et al. 1995). The ELL contains multiple topographically organized maps of the fish's body surface (Shumway 1989a). Electrosensory images that appear as spatially localized voltage perturbations on the fish's skin are transformed into spatially localized patterns of neural activity in these somatotopic maps. In general, ELL principal neurons have phasic response properties and center-surround spatial receptive field properties, with both on-center (Etype) and off-center (I-type) varieties (Bastian 1981b). They filter the incoming electrosensory image data with spatiotemporal filtering properties that vary across the different maps (Shumway 1989b). The ELL projects to the midbrain torus semicircularis, which is analogous to the mammalian inferior colliculus, as well as to the dorsal preeminential nucleus, which is part of an important electrosensory feedback loop. The ELL and associated feedback circuits are thought to play an important role in electrosensory image analysis (Rasnow 1996; Berman and Maler 1999; Lewis and Maler 2001). The torus projects to the optic tectum, which has been shown to be a site of convergence for visual and electrosensory representations of electrolocation targets (Bastian 1982), which would be useful under low-light conditions. 


\subsection{Multiple Maps in the Gymnotiform ELL}

As shown in Figure 11.7A, the gymnotiform ELL contains four subdivisions, each with its own somatotopic map of the electroreceptive periphery (Heiligenberg and Dye, 1982; Shumway 1989a, b; Metzner and Juranek 1997). The medial map is devoted to processing information from the ampullary system, which detects low-frequency electric fields of extrinsic origin. The three lateral maps are devoted to processing information from the tuberous system which is tuned to high frequencies near the fish's own EOD frequency. Each tuberous afferent trifurcates as it reaches the ELL, providing each of the three tuberous maps with essentially identical input. Amplitude and phase information is processed in parallel by separate components of the ELL circuitry in each map. In the amplitude pathway, there is a tradeoff between sensitivity and spatial resolution across the three maps, with the lateral map having the highest degree of convergence from electroreceptors and exhibiting the best sensitivity to weak stimuli, but having poor spatial resolution. In contrast, the centromedial map has good spatial resolution but low sensitivity. The lateral map has been shown to play a key role in electrocommunication behavior and the centromedial map

A
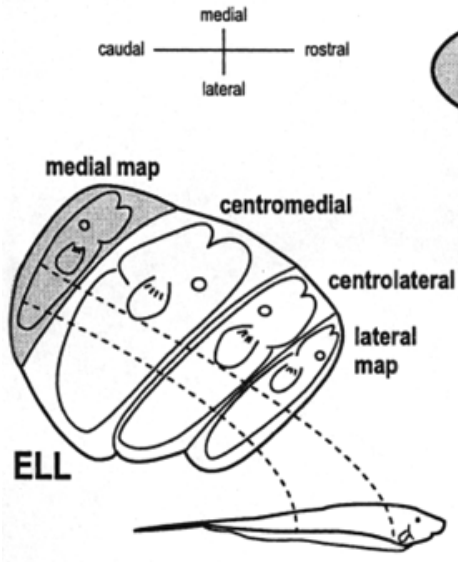
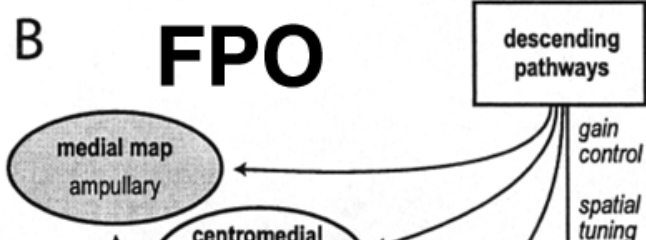

spatial
tuning

temporal

tuning

high resolution

low sensitivity

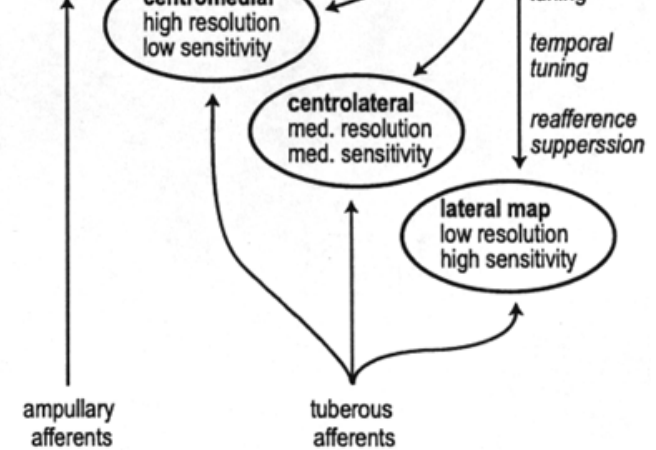

FIGURE 11.7. Schematic of the gymnotiform ELL as a multiresolution adaptive filter array. (A) Dorsoventral projection of the right ELL showing the four somatotopic maps of ampullary (gray) and tuberous (unshaded) electrosensory input. (Modified from Heiligenberg and Dye 1982.) (B) Each map processes the primary afferent input with differing resolution and sensitivity characteristics. Descending signals provide gain control, spatiotemporal tuning, and suppression of reafferent background noise. 
is necessary and sufficient for eliciting the jamming avoidance response (Metzner and Juranek 1997; Hopkins, Chapter 10).

\subsection{Distance Estimation Using Multiple Maps}

Behavioral studies have shown that mormyrid weakly electric fish can measure target distance irrespective of size, shape, or electrical conductivity of the target (von der Emde et al. 1998; von der Emde 1999). In principle, the distance to an electrolocation target can be estimated by combining a measurement of the peak amplitude of an electric image with a measurement of image width (Rasnow 1996) or maximum slope (von der Emde et al. 1998). Modeling studies have shown that topographic maps with broad spatial tuning are better for estimating image amplitude and maps with narrow tuning are better for estimating image width (Lewis and Maler 2001). Distance estimation can therefore be carried out more accurately using two maps with differing spatial resolution than with a single map (Lewis and Maler 2001). Multiple maps in the ELL may thus allow the encoding of additional sensory features, such as object distance, more efficiently than could be accomplished using a single map.

\subsection{Stimulus Coding and Feature Extraction}

Primary electrosensory afferents that project to the gymnotiform ELL fire at high rates (several hundred Hertz) and faithfully encode temporal modulations of the input signal. In contrast, the output neurons of the ELL fire at lower rates (tens of Hertz) and behave more like feature detectors under many circumstances (Gabbiani et al. 1996; reviewed in Gabbiani and Metzner 1999). There are many factors influencing the coding efficiency and feature extraction performance of ELL neurons. These performance measures depend on the functional properties of the neuron under investigation (e.g., E type versus I type) and the ELL segment in which it is found (Metzner et al. 1998), as well as on the spatial structure (e.g., local versus global) of the stimulus (Bastian et al. 2002; Chacron et al. 2003; Doiron et al. 2003). In many cases the presence of temporal features is best signaled by short bursts of spikes rather than by isolated spikes (Metzner et al. 1998). Target-related coincident activity across multiple neurons can further improve the extraction of behaviorally relevant stimulus features. Simultaneous recordings from pairs of ELL neurons have demonstrated that coincident spikes within a time window of a few milliseconds performed even better as feature detectors than bursts from single neurons. This result suggests that coincident spikes from multiple neurons can be considered as "distributed bursts" (Krahe et al. 2002). Electrophysiological studies coupled with computational models have helped elucidate the key biophysical mechanisms underlying oscillatory and burst discharge mechanisms in the ELL (Turner and Maler 1999; Doiron et al. 2001a, b, 2002, 2003). 


\subsection{Descending Control of Gain and Spatiotemporal Filtering Characteristics}

The gymnotiform ELL receives extensive descending input, which far surpasses the primary afferent input in terms of total number of synapses. These descending pathways carry various types of contextual information that could be useful in adaptive signal processing, including electrosensory feedback signals, sensory signals from other modalities, proprioceptive signals related to body posture, and efference copy signals related to outgoing motor commands (Kawasaki, Chapter 9). Lesioning the descending pathway has been shown to influence both the gain and spatiotemporal response properties of pyramidal cells in the ELL. Descending pathways interact with several classes of inhibitory interneurons in the ELL which are thought to selectively affect specific filtering functions, including gain, temporal adaptation, size of receptive field center, and size of receptive field surround. As shown in Figure 11.7B, the ELL can thus be visualized as a bank of adaptive spatiotemporal filters. It has been suggested that the different types of sensory processing carried out in the ELL-adaptive gain control, spatial filtering, temporal filtering, reafference suppression, and common mode rejection - are likely to be representative of a class of canonical signal processing strategies employed in sensory systems in general (reviewed in Berman and Maler 1999).

\subsection{Generation and Subtraction of Sensory Expectation}

A number of studies have demonstrated that the neural circuitry of the ELL plays an important role in suppressing certain "expected" components of incoming electrosensory information, particularly those associated with sensory reafference in which an animal's motor actions have predictable sensory consequences (Bastian and Zakon, Chapter 8). Adaptive reafference suppression in the ELL was first discovered in mormyrid pulse-type fish, in which the neural circuitry adaptively constructs a "negative image" of the expected pattern of sensory input over a period of several minutes (reviewed in Bell 2001). Over the past several years the neural mechanisms underlying this phenomenon in the ELL have been worked out in considerable detail, leading to the present view that the negative image is formed by anti-Hebbian mechanisms operating at descending pathway synapses (Bastian and Zakon, Chapter 8). Modeling studies have helped explain the functional benefits of temporal asymmetry in the synaptic modification rule (Roberts 1999; Roberts and Bell 2000). This negative image mechanism is responsible adaptively suppressing reafferent signals associated with tail bending in gymnotiform weakly electric fish (Bastian 1995; Bastian and Zakon, Chapter 10). 


\section{Directions for Future Research}

Although much progress has been made, many gaps remain in our understanding of electrolocation. One fruitful area for future investigation involves a better characterization of the statistical properties of natural electrosensory scenes. Compared to other sensory modalities, such as vision and audition, we have much less knowledge of sensory signal and background characteristics under naturalistic conditions. There is also a gap in our knowledge related to a lack of behavioral data from the field. Almost all electrolocation studies to date have been carried out in laboratory settings. Because weakly electric fish are nocturnal, live in turbid waters, and are found in relatively remote locations, the technical challenges of recording and analyzing electrolocation behavior in the field are significant. Consequently, we know very little about the natural foraging and feeding strategies that these animals use in their natural habitats.

On the electrophysiology front, we need to learn more about neural activity patterns while the animal is actively performing an electrolocation task. Almost all in vivo electrophysiology related to electrolocation has been carried out on restrained animals that are not engaged in any sort of behavioral task. (An exception is the jamming avoidance response, in which a complete behavioral loop, from sensory input to motor output, can be studied in a restrained animal.) Because the gain and spatiotemporal filtering properties of sensory neurons can be modulated at early levels of sensory processing, it is likely that neurons at the level of the ELL and higher will show task-dependent response properties. Investigating adaptive aspects of the neural algorithms underlying target detection, characterization, and localization may require that the fish be actively engaged in an electrolocation task while neural responses are being probed. Ideally, one would like to be able to record and monitor neural activity from multiple neurons in a freely swimming fish. Again, the technical challenges are significant, but developing multineuron chronic recording and telemetry capabilities could dramatically advance our understanding of the adaptive aspects electrolocation.

Another major area for future investigation lies on the motor side of the electrolocation problem. The sensory processing side of the task has been the primary focus of attention for several decades, whereas little work has been done on motor aspects related to active repositioning of the sensor array and target interception. The control of the ribbon fin in knifefish is a particularly appealing problem from a number of perspectives. Although there has been some work on the biomechanical and hydrodynamic aspects of gymnotiform locomotion, little is known about the neural control of the traveling waves on the ribbon fin. Also, little is known about the central representation of the motor space and target location in the nervous system. It would also be interesting to explore what sorts of motor planning and control strategies the fish might use to optimize the acquisition of sensory information from the environment. 


\section{Summary}

Weakly electric fish can detect, localize, and characterize sensory targets in their environment using electrosensory cues. Passive electrolocation relies on detection and analysis of the intrinsic electric fields generated by the target object, while active electrolocation relies on target-induced perturbations in the fish's self-generated electric field. The active mode allows the fish to detect a broader spectrum of target objects, because it requires only that the electrical impedance of the target differ from the surrounding water. The active electric sense has a limited working range of a few centimeters for detecting small prey and about one body length for larger objects. Fish have been shown to be capable of discriminating differences in size, shape, distance, and impedance of target objects.

Some species of weakly electric fish emit brief pulse-type electric organ discharges with a broad frequency spectrum, while others have continuous wavetype discharges with the energy concentrated at the fundamental frequency and the first few harmonics. In contrast to certain echolocating bats, weakly electric fish do not appear to make adaptive adjustments to the amplitude or frequency of their discharge as they approach a target.

The spatial structure of the fish's electric field is approximately dipolar, although the near field is considerably more complex. The presence of a target object induces a local perturbation that is proportional to the strength of the fish's electric field at the target location. For small spherical targets, the perturbation can be accurately modeled as an induced dipole. When mapped onto the receptor surface, the voltage perturbation gives rise to an electrosensory image with a Gaussian-like intensity distribution. Peak image intensity falls rapidly with increasing target distance (approximately as $r^{-3}$ in the near field), and image width is roughly equal to target distance. Computer modeling has been an important tool for understanding the spatial structure of the fish's electric field, the effects of object perturbations, and the spatiotemporal properties of electrosensory images at the receptor surface.

Image intensity scales linearly with the target's volume and electrical contrast-a measure of the relative difference in impedance between the target and the water. Weakly electric fish can assess complex impedance properties by analyzing amplitude and phase information, which is typically encoded by separate classes of primary afferent nerve fibers. Modeling studies have been pivotal in characterizing the response dynamics and coding properties of primary afferents, providing insights into how transdermal voltage patterns are converted into spatiotemporal patterns of spike activity in the CNS.

Primary electrosensory afferents project to the hindbrain ELL. In gymnotiforms, primary afferents faithfully encode the temporal structure of the input signal, while ELL pyramidal neurons seem to behave more like feature detectors. The ELL is divided into multiple somatotopic maps with differing tradeoffs between sensitivity and spatial resolution. Descending pathways are involved in modulating the gain and spatiotemporal filtering properties of ELL pyramidal 
cells and in suppressing reafferent backgrounds. The functional role of multiple maps in electrolocation is not well understood. One hypothesis is that different maps may subserve different phases of electrolocation behavior, with one map playing a key role during detection and others during localization and characterization. Modeling studies suggest that target distance could be estimated more accurately using multiple maps than with a single map. Electrophysiological models are helping illuminate the biophysical basis of neural information processing mechanisms in the ELL, such as gain control and burst coding.

Exploring principles of electrolocation at a deeper level will require an integrative approach that combines aspects of anatomy, physiology, behavior, sensory ecology, and neural information processing principles. A solid foundation has been laid, as evidenced by the wealth of information presented in this volume, but much more remains to be discovered before we fully understand this fascinating sensory capability.

Acknowledgments. This work was supported by grants from NSF (IBN0078206) and NIMH (R01-MH49242).

\section{References}

Assad C (1997) Electric field maps and boundary element simulations of electrolocation in weakly electric fish. PhD thesis, California Institute of Technology, Pasadena, CA.

Assad C, Rasnow B, Stoddard PK (1999) Electric organ discharges and electric images during electrolocation. J Exp Biol 202:1185-1193.

Bacher M (1983) A new method for the simulation of electric fields generated by electric fish and their distortions by objects. Biol Cybern 47:51-58.

Bass AH (1986) Electric organs revisited: evolution of a vertebrate communication and orientation organ. In: Bullock TH, Heiligenberg W (eds), Electroreception. New York: John Wiley \& Sons, pp. 13-70.

Bastian J (1981a) Electrolocation I. How the electroreceptors of Apteronotus albifrons code for moving objects and other electrical stimuli. J Comp Physiol 144:465-479.

Bastian J (1981b) Electrolocation II. The effects of moving objects and other electrical stimuli on the activities of two categories of posterior lateral line lobe cells in Apteronotus albifrons. J Comp Physiol 144:481-494.

Bastian J (1982) Vision and electroreception integration of sensory information in the optic tectum of the weakly electric fish Apteronotus albifrons. J Comp Physiol 147: 287-298.

Bastian J (1995) Pyramidal-cell plasticity in weakly electric fish: a mechanism for attenuating responses to reafferent electrosensory inputs. J Comp Physiol A 176:63-78.

Bastian J (2003) Electrolocation. In: Arbib M (ed), The Handbook of Brain Theory and Neural Networks. Cambridge, MA: MIT Press, pp. 391-398.

Bastian J, Chacron MJ, Maler L (2002) Receptive field organization determines pyramidal cell stimulus-encoding capability and spatial stimulus selectivity. J Neurosci 22:45774590.

Bell CC (1990) Mormyromast electroreceptor organs and their afferent fibers in Mor- 
myrid fish III. Physiological differences between two morphological types of fibers. J Neurophysiol 63:319-332.

Bell CC (2001) Memory-based expectations in electrosensory systems. Curr Opin Neurobiol 11:481-487.

Berman NJ, Maler L (1999) Neural architecture of the electrosensory lateral line lobe: adaptations for coincidence detection, a sensory searchlight and frequency-dependent adaptive filtering. J Exp Biol 202:1243-1253.

Brandman R, Nelson ME (2002) A simple model of long-term spike train regularization. Neural Comput 14:1575-1597.

Budelli R, Caputi AA (2000) The electric image in weakly electric fish: perception of objects of complex impedance. J Exp Biol 203:481-492.

Caputi AA, Budelli R, Grant K, Bell CC (1998) The electric image in weakly electric fish-physical images of resistive objects in Gnathonemus petersii. J Exp Biol 201: 2115-2128.

Carr CE (1986) Time coding in electric fish and barn owls. Brain Behav Evol 28:122133.

Carr CE, Maler L, Sas E (1982) Peripheral organization and central projections of the electrosensory nerves in gymnotiform fish. J Comp Neurol 211:139-153.

Chacron MJ, Longtin A, St-Hilaire M, Maler L (2000) Suprathreshold stochastic firing dynamics with memory in P-type electroreceptors. Phys Rev Lett 85:1576-1579.

Chacron MJ, Longtin A, Maler L (2001) Negative interspike interval correlations increase the neuronal capacity for encoding time-dependent stimuli. J Neurosci 21:5328-5343.

Chacron MJ, Doiron B, Maler L, Longtin A, Bastian J (2003) Non-classical receptive field mediates switch in a sensory neuron's frequency tuning. Nature 423:77-81.

Doiron B, Longtin A, Turner RW, Maler L (2001a) Model of gamma frequency burst discharge generated by conditional backpropagation. J Neurophysiol 86:1523-1545.

Doiron B, Longtin AAE, Berman N, Maler L (2001b) Subtractive and divisive inhibition: effect of voltage-dependent inhibitory conductances and noise. Neural Comput 13: 227-248.

Doiron B, Laing C, Longtin A, Maler L (2002) Ghostbursting: a novel neuronal burst mechanism. J Comput Neurosci 12:5-25.

Doiron B, Chacron MJ, Maler L, Longtin A, Bastian J (2003) Inhibitory feedback required for network oscillatory responses to communication but not prey stimuli. Nature 421:539-543.

Gabbiani F, Metzner W (1999) Encoding and processing of sensory information in neuronal spike trains. J Exp Biol 202:1267-1279.

Gabbiani F, Metzner W, Wessel R, Koch C (1996) From stimulus encoding to feature extraction in weakly electric fish. Nature 384:564-567.

Heiligenberg W (1975) Theoretical and experimental approaches to spatial aspects of electrolocation. J Comp Physiol 103:247-272.

Heiligenberg W (1977) Principles of electrolocation and jamming avoidance studies of brain function. Vol 1. Berlin: Springer-Verlag.

Heiligenberg W, Dye J (1982) Labeling of electroreceptive afferents in a gymnotoid fish by intracellular injection of horseradish peroxidase: the mystery of multiple maps. J Comp Physiol 148:287-296.

Hopkins CD (1976) Stimulus filtering and electroreception: tuberous receptors in three species of gymnotoid fish. J Comp Physiol A 111:171-207.

Hoshimiya N, Shogen K, Matsuo T, Chichibu S (1980) The Apteronotus electric organ

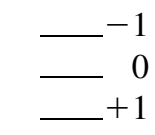


discharge field waveform and electric organ discharge field simulation. J Comp Physiol 135:283-290.

Jackson JD (1999) Classical Electrodynamics, 3rd edit. New York: John Wiley \& Sons.

Kashimori Y, Goto M, Kambara T (1996) Model of P- and T-electroreceptors of weakly electric fish. Biophys J 70:2513-2526.

Knudsen EI (1975) Spatial aspects of the electric fields generated by weakly electric fish. J Comp Physiol 99:103-118.

Krahe R, Kreiman G, Gabbiani F, Koch C, Metzner W (2002) Stimulus encoding and feature extraction by multiple sensory neurons. J Neurosci 22:2374-2382.

Kreiman G, Krahe R, Metzner W, Koch C, Gabbiani F (2000) Robustness and variability of neuronal coding by amplitude-sensitive afferents in the weakly electric fish Eigenmannia. J Neurophysiol 84:189-204.

Lannoo MJ, Lannoo SJ (1993) Why do electric fishes swim backwards? An hypothesis based on Gymnotiform foraging behavior interpreted through sensory constraints. En. Bio. Fishes 36:157-165.

Lewis JE, Maler L (2001) Neuronal population codes and the perception of object distance in weakly electric fish. J Neurosci 21:2842-2850.

Lissmann HW (1958) On the function and evolution of electric organs in fish. J Exp Biol 35:156-191.

Lissman HW, Machin KE (1958) The mechanism of object location in Gymnarchus niloticus and similar fish. J Exp Biol 35:451-486.

MacIver MA, Nelson ME (2000) Body modeling and model-based tracking for neuroethology. J Neurosci Methods 95:133-143.

MacIver MA, Sharabash NM, Nelson ME (2001) Prey-capture behavior in gymnotid electric fish: motion analysis and effects of water conductivity. J Exp Biol 204:543557.

Metzner W, Juranek J (1997) A sensory brain map for each behavior. Proc Natl Acad Sci USA 94:14798-14803.

Metzner W, Koch C, Wessel R, Gabbiani F (1998) Feature extraction by burst-like spike patterns in multiple sensory maps. J Neurosci 18:2283-2300.

Moller P (1995) Electric Fishes: History and Behavior. London: Chapman and Hall.

Montgomery J, Coombs S, Conley RA, Bodznick D (1995) Hindbrain sensory processing in lateral line, electrosensory and auditory systems: a comparative overview of anatomical and functional similarities. Audit Neurosci 1:207-231.

Moortgat KT, Keller CH, Bullock TH, Sejnowski TJ (1998) Submicrosecond pacemaker precision is behaviorally modulated: the gymnotiform electromotor pathway Proc Natl Acad Sci USA 95:4684-4689

Nelson ME, MacIver MA (1999) Prey capture in the weakly electric fish Apteronotus albifrons: sensory acquisition strategies and electrosensory consequences. J Exp Biol 202:1195-1203.

Nelson ME, Xu Z, Payne JR (1997) Characterization and modeling of P-type electrosensory afferent responses to amplitude modulations in a wave-type electric fish. J Comp Physiol A 181:532-544.

Nelson ME, MacIver MA, Coombs S (2002) Modeling electrosensory and mechanosensory images during the predatory behavior of weakly electric fish. Brain Behav Evol 59:199-210.

Rasnow B (1996) The effects of simple objects on the electric field of Apteronotus. J Comp Physiol A 178:397-411. 
Rasnow B, Bower JM (1996) The electric organ discharges of the Gymnotiform fishes: I. Apteronotus leptorhynchus. J Comp Physiol A 178:383-396.

Ratnam R, Nelson ME (2000) Nonrenewal statistics of electrosensory afferent spike trains: implications for the detection of weak sensory signals. J Neurosci 20:66726683.

Roberts PD (1999) Computational consequences of temporally asymmetric learning rules: I. Differential Hebbian learning. J Comput Neurosci 7:235-246.

Roberts PD, Bell CC (2000) Computational consequences of temporally asymmetric learning rules: II. Sensory image cancellation. J Comput Neurosci 9:67-83.

Scheich H, Bullock TH, Hamstra RH (1973) Coding properties of two classes of afferent nerve fibers: high-frequency electroreceptors in the electric fish, Eigenmannia. J Neurophysiol 36:39-60.

Shumway CA (1989a) Multiple electrosensory maps in the medulla of weakly electric Gymnotiform fish I. Physiological differences. J Neurosci 9:4388-4399.

Shumway CA (1989b) Multiple electrosensory maps in the medulla of weakly electric Gymnotiform fish II. Anatomical differences. J Neurosci 9:4400-4415.

Turner RW, Maler L (1999) Oscillatory and burst discharge in the apteronotid electrosensory lateral line lobe. J Exp Biol 202:1255-1265.

von der Emde G (1999) Active electrolocation of objects in weakly electric fish. J Exp Biol 202:1205-1215.

von der Emde G, Bleckmann H (1998) Finding food: senses involved in foraging for insect larvae in the electric fish Gnathonemus petersii. J Exp Biol 201:969-980.

von der Emde G, Schwarz S, Gomez L, Budelli R, Grant K (1998) Electric fish measure distance in the dark. Nature 395:890-894.

Wessel R, Koch C, Gabbiani F (1996) Coding of time-varying electric field amplitude modulations in a wave-type electric fish. J Neurophysiol 75:2280-2293.

Wilkens LA, Russell DF, Pei X,Gurgens C (1997) The paddlefish rostrum functions as an electrosensory antenna in plankton feeding. Proc Soc Lond B 264:1723-1729.

Xu Z, Payne JR, Nelson ME (1996) Logarithmic time course of sensory adaptation in electrosensory afferent nerve fibers in a weakly electric fish. J Neurophysiol 76:20202032.

Yager DD, Hopkins CD (1993) Directional characteristics of tuberous electroreceptors in the weakly electric fish, Hypopomus (Gymnotiformes). J Comp Physiol A 143:401414. 